\title{
Magnetic resonance-guided focused ultrasound thalamotomy for essential tremor: a 5-year single-center experience
}

\author{
*Alon Sinai, PhD, ${ }^{1}$ Maria Nassar, MD, ${ }^{2}$ Ayelet Eran, MD,, 3 Marius Constantinescu, MD, ${ }^{3}$ \\ Menashe Zaaroor, MD, DSc, ${ }^{1,4}$ Elliot Sprecher, PhD, ${ }^{2}$ and llana Schlesinger, MD ${ }^{2,4}$ \\ Departments of ${ }^{1}$ Neurosurgery, ${ }^{2}$ Neurology, and ${ }^{3}$ Radiology, Rambam Health Care Campus, Haifa; and ${ }^{4}$ Technion Faculty of \\ Medicine, Haifa, Israel
}

\begin{abstract}
OBJECTIVE The authors report their experience in treating patients suffering from medication-resistant essential tremor (ET) with MR-guided focused ultrasound (MRgFUS) thalamotomy over a 5-year period.
\end{abstract}

METHODS Forty-four ET patients treated with unilateral MRgFUS ventral intermediate nucleus (VIM) thalamotomy were assessed using the Clinical Rating Scale for Tremor (CRST) score and the Quality of Life in Essential Tremor Questionnaire (QUEST) over a 5-year span.

RESULTS Tremor was significantly improved immediately following MRgFUS in all patients and ceased completely in 24 patients. CRST scores in the treated hand at baseline (median 19; range 7-32, 44 patients) improved by a median of 16 at 1 month (44 patients; $p<0.0001), 17$ at 6 months (31 patients; $p<0.0001), 15$ at 1 year (24 patients; $p<0.0001), 18$ at 2 years (15 patients; $p<0.0001), 19$ at 3 years, (10 patients; $p<0.0001), 21$ at 4 years ( 6 patients; $p<0.01$ ), and 23 at 5 years (2 patients, significance not tested). Return of tremor that impacted activities of daily living was reported in 5 patients (11\%). QUEST scores showed significant improvement, with median change of 35 points $(p<0.0001 ; 44$ patients) at 1 month, 33 ( $p<0.0001 ; 31$ patients) at 6 months, 27 ( $p<0.0001 ; 24$ patients) at 1 year, 26 ( $p<0.001 ; 15$ patients) at 2 years, 25 ( $p<0.001 ; 10$ patients) at 3 years, 33 ( $p<0.001 ; 6$ patients) at 4 years, and 28 (significance not tested, 2 patients) at 5 years. Adverse events after the procedure were reversible in all but 5 patients $(11 \%)$.

CONCLUSIONS MRgFUS thalamotomy for ET is an effective and safe procedure that provides long-term tremor relief and improvement in quality of life even in patients with medication-resistant disabling tremor. Additional studies with a larger group of patients is needed to substantiate these favorable results.

https://thejns.org/doi/abs/10.3171/2019.3.JNS19466

KEYWORDS essential tremor; MR-guided focused ultrasound; treatment; surgery; thalamotomy; functional neurosurgery

$\mathrm{U}$ NILATERAL MR-guided focused ultrasound (MRgFUS) ventral intermediate nucleus (VIM) thalamotomy for treatment of medically refractory and debilitating tremor has been CE approved since 2013 and was FDA approved for treatment of essential tremor (ET) in July 2016. The treatment involves multiple sonications, starting with low-energy sonications with gradual raising of the temperature until a therapeutic response is observed without undesired side effects. ${ }^{16}$ Such treatments performed multiple times will eventually sculpt the final lesion in the contralateral VIM. ${ }^{3,6,10,14}$ Since the thalamic VIM nucleus boundaries are not visible on MRI, this procedure is dependent on meticulous, tenth-of-a-millimeter movements of the focal point from one sonication to another, with continuous feedback from the patient throughout the entire procedure. ${ }^{5,10,12}$ Reports by our group and others suggest that MRgFUS thalamotomy is effective, with a good safety profile. However, information regarding safety and sustainability of tremor relief over time is currently limited. ${ }^{2,4}$ We report our experience over a span of 5

ABBREVIATIONS CRST = Clinical Rating Scale for Tremor; ET = essential tremor; LOWESS = locally weighted scatterplot smoothing; MRgFUS = MR-guided focused ultrasound; PD = Parkinson's disease; QUEST = Quality of Life in Essential Tremor Questionnaire; SDR = skull density ratio; VIM = ventral intermediate nucleus. SUBMITTED February 22, 2019. ACCEPTED March 29, 2019.

INCLUDE WHEN CITING Published online July 5, 2019; DOI: 10.3171/2019.3.JNS19466.

${ }^{*}$ A.S. and M.N. contributed equally to this work. 
years with unilateral VIM thalamotomy using MRgFUS in patients with medication-resistant ET.

\section{Methods \\ Patients}

Patients with disabling medication-resistant ET were treated with MRgFUS. VIM thalamotomy was performed contralateral to the patient's hand preference. All patients were offered either deep brain stimulation or MRgFUS and preferred MRgFUS as their treatment of choice.

The diagnosis of ET was confirmed according to accepted criteria by a neurologist specializing in movement disorders (I.S.). Medication resistance to at least 2 medications at appropriate doses was verified by a movement disorders neurologist (I.S.). Refractory tremor was considered as disabling tremor after ample treatment trials.

All patients had no contraindications for the procedure, including but not limited to significant cognitive decline, current anticoagulant or antiaggregant therapy, brain tumors, vascular malformations, significant unstable medical conditions, and contraindications for MR, including claustrophobia.

The study was approved by the institutional review board.

\section{Assessments}

Tremor was assessed using the Clinical Rating Scale for Tremor (CRST) score (ranging from 0-160 points, higher scores indicating greater disability). ${ }^{13}$ A clinically significant tremor was defined as a score of 2 or more on the postural or action item of the CRST (ranging from 0 to 4), as well as substantial disability in the performance of at least 2 daily activities from the disability subsection of the scale. We also calculated CRST scores in the treated hand as well (maximum overall score, 32 ).

Quality of life in ET patients was measured by the Quality of Life in Essential Tremor Questionnaire (QUEST) (scores ranging from 0-120; higher scores indicate lower quality of life)..$^{15}$

Adverse events were rated according to the ClavienDindo criteria (range 1-5, higher score representing a more severe event). ${ }^{7}$

\section{Imaging and Treatment Parameters}

All patients underwent preprocedural MRI and CT. All MRI studies were performed using a 3-Tesla system (MR750, GE). The following pulse sequences were performed in all studies: fast spin echo (FSE) T2 in axial, sagittal, and coronal planes; axial spoiled gradient recalled (SPGR) T1, SWI, FLAIR, and DTI and postcontrast SPGR T1 and FSE T1 coronal images. CT images were used to assess the ratio between the bone and the bone marrow of the skull, the skull density ratio (SDR), using data from the Exablate console. ${ }^{4}$ An SDR of 0.3 or above was considered suitable for treatment. Information regarding skull volume available for sonication, available skull surface, and number of elements used during treatment were gathered from the Exablate console as well. Patients underwent MRI 1 day and 1 week following treatment. Lesion volume and lesion volume with edema were measured from T2 FSE images using Carestream Vue Pacs (Carestream Health Inc., 2014).

\section{Statistical Analysis}

Differences in tremor and quality of life scores at each landmark follow-up time point were calculated as simple individual differences of the score at that time minus the pretreatment score. Percent changes in tremor and quality of life were calculated as the difference in the tremor scores after versus before the treatment, divided by the score before the procedure and multiplied by 100 . Percent change was calculated for the total CRST score and for the CRST score in the treated hand.

Most patients evidenced $100 \%$ or near total improvement of tremor for much of the follow-up period, resulting in kurtotic and skewed distributions of change scores. Therefore, medians and ranges are reported when necessary, along with appropriate nonparametric statistical analyses. These included Wilcoxon signed-rank tests of change scores at each follow-up time (without adjustment for multiplicity), and for relationships of factors related to degree of change, nonparametric Spearman correlations rather than Pearson correlations. These correlations are categorized as weak when below 0.5 , moderate when between 0.5 to 0.75 , and strong when above 0.75 . Graphic analysis of interrelationships of continuous interval or ratio level data included locally weighted scatterplot smoothing (LOWESS) curves. Prediction of adverse events was performed using logistic regression or likelihood ratio chi-square for continuous or nominal predictors, respectively. Attempts to determine a threshold for acceptable SDR magnitude for successful treatment were performed using logistic regression and ROC (receiver operating characteristic) analysis. We attempted to develop predictive models for treatment outcome based on patient and treatment characteristics, using stepwise methods (while controlling for correlated potential predictors), but the current number of patients in follow-up did not provide sufficient statistical power.

JMP software (SAS Institute) was utilized for statistical analyses.

\section{Results \\ Patients}

Between November 2013 and November 2018, 48 patients with severe refractory tremor underwent MRgFUS thalamotomy using a focused ultrasound system $(650-\mathrm{kHz}$ system, ExAblate, Insightec) for VIM ablation. Of these patients, 4 patients developed Parkinson's disease (PD) many years after ET, and thus these patients were diagnosed as suffering from ET-PD. Since these patients had different symptoms and signs, mostly related to their PD, they were excluded from this cohort. Of the remaining 44 ET patients, 27 were male. Thirty-eight patients were right handed. Treatment was aimed to alleviate tremor in the dominant hand in 41 patients. The median age of patients was 70.5 years (range $63-87$ years). The mean disease duration was $16.3 \pm 10.4$ (range $1-30$, median, 13.5 years). Median follow-up after MRgFUS thalamotomy was 12 months, with a range of $1-60$ months. As expected in a clinical setting, some patients were lost to follow-up. 
TABLE 1. Assessment scores before and after MRgFUS treatment

\begin{tabular}{lcccccccc}
\hline & & \multicolumn{5}{c}{ Months of Follow-Up } \\
\cline { 3 - 8 } & Before Treatment & 1 & 6 & 12 & 24 & 36 & 48 & 60 \\
\hline No. of patients & 44 & 44 & 31 & 24 & 15 & 10 & 6 & 2 \\
\hline CRST score, treated hand & $19.0(7-32)$ & $0.0(0-12)$ & $3.0(0-17)$ & $4.0(0-20)$ & $4.0(0-22)$ & $3.5(0-16)$ & $5.0(0-21)$ & $3.0(2-4)$ \\
\hline$\quad$ Delta & & $-16^{* * *}$ & $-17^{* * *}$ & $-15^{* * *}$ & $-18^{* * *}$ & $-19^{* * *}$ & $-21^{* *}$ & -23 \\
\hline CRST score, total & $46.0(16-74)$ & $12.0(0-38)$ & $14.5(3-43)$ & $18.0(3-48)$ & $11.0(4-57)$ & $16.0(9-57)$ & $14.0(6-74)$ & $8.0(6-10)$ \\
\hline$\quad$ Delta & & $-29^{* * *}$ & $-31^{* * *}$ & $-27^{* * *}$ & $-30^{* * *}$ & $-33^{*}$ & $-39^{*}$ & -49 \\
\hline QUEST score & $41.5(15-93)$ & $5.5(0-86)$ & $8.0(0-86)$ & $14.0(0-89)$ & $15.0(0-78)$ & $15.5(8-59)$ & $14.5(4-28)$ & $11.0(6-16)$ \\
\hline Delta & & $-35^{* * *}$ & $-33^{* * *}$ & $-27^{* * *}$ & $-26^{* *}$ & $-25^{* *}$ & $-33^{* *}$ & -28 \\
\hline
\end{tabular}

Delta values are the median pairwise differences (follow-up - before treatment) of the score for each patient available at follow-up relative to before-treatment reading; negative scores indicate improvement. Wilcoxon signed-rank tests were performed on deltas. Only 2 patients completed follow-up at 60 months; their delta significance was not tested. ${ }^{* * *} p<0.0001,{ }^{* *} p<0.001$, and ${ }^{*} p<0.05$ compared with baseline.

\section{Assessments}

Tremor was significantly improved immediately following MRgFUS thalamotomy in all patients. Twentyfour patients had complete cessation of tremor in the treated hand immediately following treatment. Total CRST scores and CRST scores in the treated hand before treatment were significantly decreased after treatment and remained significantly lower during follow-up. Table 1 and
Figs. 1 and 2 provide full details as to changes in CRST over follow-up and the individual patient time courses of CRST during follow-up.

Before-treatment quality of life score significantly correlated with CRST score in the hand selected for treatment $(\varrho=-0.38, p=0.010)$. QUEST scores were significantly improved after treatment relative to baseline scores, see Table 1 for changes in QUEST over time and Fig. 3 for

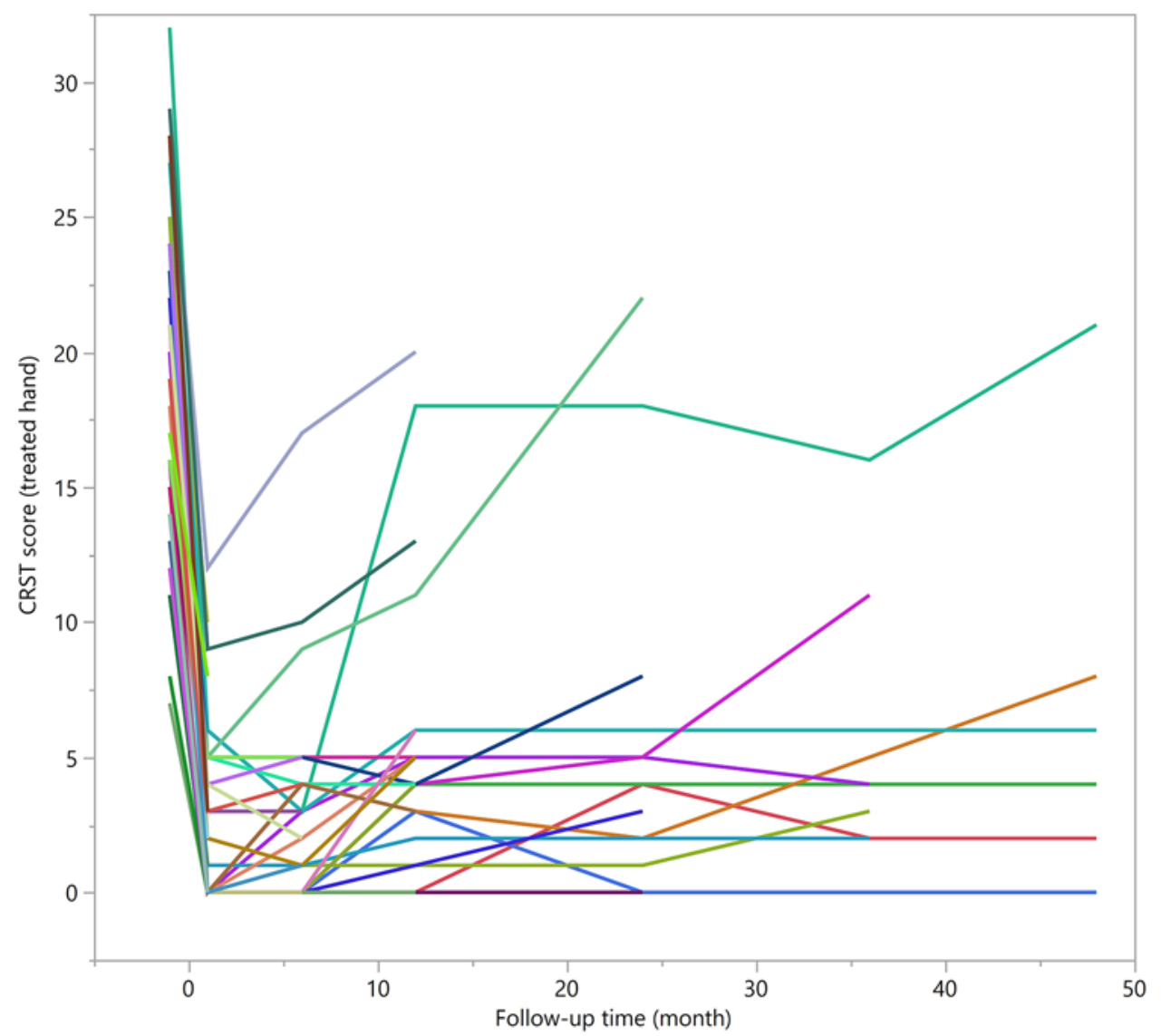

FIG. 1. CRST score of each patient in treated hand at follow-up visits, up to 48 months. Significant symptomatic improvement in tremor in the treated hand was seen (reduction in tremor score). Significant symptomatic improvement in tremor was maintained over time. Figure is available in color online only. 


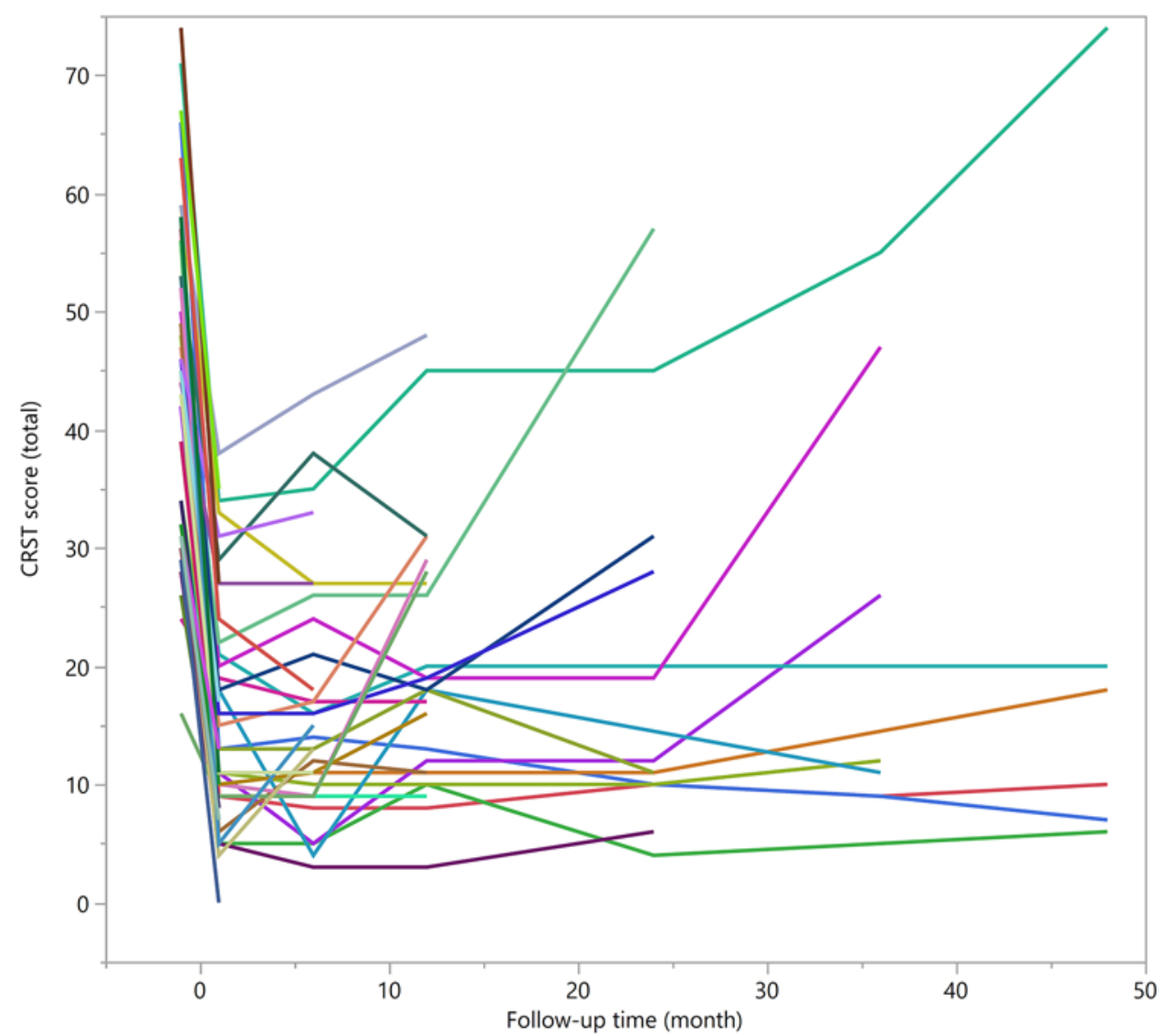

FIG. 2. Total CRST score of each patient at follow-up visits, up to 48 months. Significant symptomatic improvement in tremor was seen (reduction in tremor score). Significant symptomatic improvement was maintained over time. Figure is available in color online only.

individual patient time courses of QUEST during followup. At 1 month following treatment, 7 patients reported no burden at all on their quality of life, with a QUEST score of 0 . Some return of tremor was seen during follow-up (see Fig. 3). Tremor return had significant impact on activities of daily living in 5 patients (11\% of patients).

\section{Treatment Parameters}

Treatment parameters are detailed in Table 2.

We analyzed whether tremor severity before MRgFUS thalamotomy, as measured by CRST, affected treatment parameters and found that higher CRST scores before treatment were significantly associated with higher maximal temperatures used for ablation at treated targets $(\mathrm{Q}=$ $0.33, p=0.030$ ) but not with the number of sonications, duration of longest sonication, or maximal energy used for ablation.

The distribution of SDR calculations in our cohort is shown in Figs. 4 and 5. In 2 patients a CT protocol was used that did not enable calculation. Maximal temperature reached during treatment significantly correlated with SDR ( $\varrho=0.54, p=0.0002)$ (Fig. 4), while maximal energy attained at the end of the treatment was inversely correlated with SDR ( $\varrho=-0.36, \mathrm{p}=0.019)$ (Fig. 5).

The maximal sonication time correlated with maximal energy used during the treatment $(\varrho=0.63, p<0.0001)$.
The maximal sonication time also correlated with lesion volume and lesion volume with surrounding edema at 1 day $(\varrho=0.46, p=0.0016$, and $\varrho=0.43, p=0.0036$, respectively) and 7 days following the procedure $(\varrho=0.56, p<$ 0.0001 , and $\varrho=0.44, p=0.0031$, respectively). The length of maximum sonication time significantly correlated with number of sonications $(\mathrm{o}=0.48, \mathrm{p}=0.001)$. It should be noted that the number of sonications in our cohort had a wide range, from 9 to 36 (see Table 2). The number of sonications also correlated with maximal energy used during treatment $(\mathrm{o}=0.54, \mathrm{p}=0.0002)$. In turn, the maximal energy used during treatment correlated with the lesion volume and lesion volume with surrounding edema at 1 day ( $\varrho=0.41, p=0.0052$, and $\varrho=0.35, p=0.019$, respectively) and 7 days following the procedure $(\mathrm{Q}=0.32, \mathrm{p}=0.036$, and $\varrho=0.47, \mathrm{p}=0.0014$, respectively).

\section{Imaging}

The mean lesion volume at 1 day was $297.1 \pm 128.0$ $\mathrm{mm}^{3}$ (range $92-729 \mathrm{~mm}^{3}$, median $276.40 \mathrm{~mm}^{3}$ ), while the mean lesion volume at 1 week was $392.9 \pm 383.7 \mathrm{~mm}^{3}$ (range 50.2-2636.3 $\mathrm{mm}^{3}$, median $299.9 \mathrm{~mm}^{3}$ ). The mean lesion volume with surrounding edema at 1 day was 1459.9 $\pm 769.0 \mathrm{~mm}^{3}$ (range $259.2-3631.3 \mathrm{~mm}^{3}$, median 1239.2 $\mathrm{mm}^{3}$ ) and at 1 week was $1306.2 \pm 1204.7 \mathrm{~mm}^{3}$ (range $0-7284.9 \mathrm{~mm}^{3}$, median $1038.40 \mathrm{~mm}^{3}$ ). 


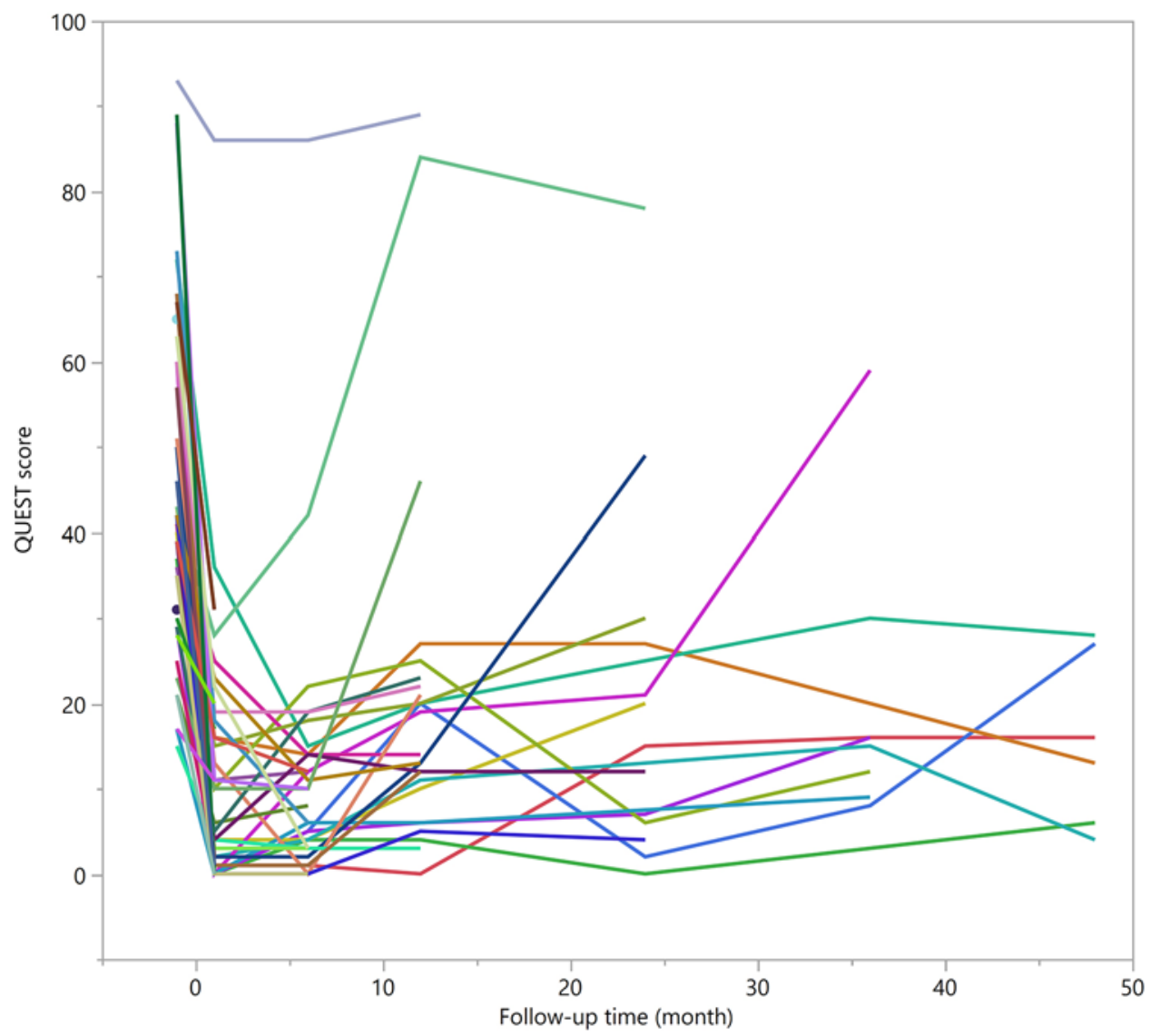

FIG. 3. QUEST score in each patient at follow-up visits, up to 48 months. Significant improvement in quality of life was seen (reduction in disability score). Significant symptomatic improvement in quality of life was maintained over time. Figure is available in color online only.

Imaging parameters including lesion volume with surrounding edema and lesion volume without surrounding edema at 1 day and at 1 week did not correlate with outcome. Maximal energy used for ablation significantly correlated with lesion volume at 1 day and 1 week $(\varrho=0.41$, $\mathrm{p}=0.0052$, and $\varrho=0.32, \mathrm{p}=0.036$, respectively) and with volume of lesion with surrounding edema at 1 day and 1 week $(\mathrm{Q}=0.35, \mathrm{p}=0.019$, and $\mathrm{Q}=0.47, \mathrm{p}<0.0014$, respectively). Maximal temperature at the site of ablation correlated only with lesion volume at 1 day $(\varrho=0.32, p=$ 0.037 ), while the number of sonications correlated with volume of lesion with surrounding edema at 1 day $(\varrho=$ $0.30, \mathrm{p}=0.046)$.

\section{Predictors of Treatment Efficacy}

We examined factors that could possibly affect treatment success as measured by the percentage of change in tremor. We found that at 1 month only SDR was significantly correlated with significant improvement in tremor ( $\varrho=0.40, p=0.0082)$. And again at 6 months, SDR was the only treatment parameter that correlated with reduced tremor $(\varrho=0.38, p=0.044)$. At 12 months, SDR was no longer significantly correlated with degree of tremor reduction $(\varrho=0.12, p=0.6)$. Since studies looking at efficacy of MRgFUS thalamotomy included patients above a certain SDR threshold, we looked for such a threshold for successful treatment in our cohort, but could not find such a threshold.

Patients' sex, age, and disease duration did not affect outcome after MRgFUS thalamotomy. Treatment parameters such as number of elements used during treatment, skull area available for sonication, skull volume available for sonication, maximal energy used during treatment, maximal temperature measured at treated brain target at the end of treatment, and maximal sonication time also did not significantly affect results of MRgFUS thalamotomy. Only the number of sonications used during treatment significantly correlated with the percent of change in

TABLE 2. Treatment parameters of MRgFUS

\begin{tabular}{lc}
\hline \multicolumn{1}{c}{ Parameters } & Median (range) \\
\hline SDR & $0.47(0.31-0.67)$ \\
\hline Available skull vol, $\mathrm{cm}^{3}$ & $819.0(654.8-978.6)$ \\
\hline Available skull area, $\mathrm{cm}^{2}$ & $321.6(268.7-365.4)$ \\
\hline No. of elements & $888(740-963)$ \\
\hline Maximal sonication time, sec & $13.0(10-32)$ \\
\hline No. of sonications & $19.5(9-36)$ \\
\hline Maximal energy, $\mathrm{J}$ & $12,077(6000-35,500)$ \\
\hline
\end{tabular}




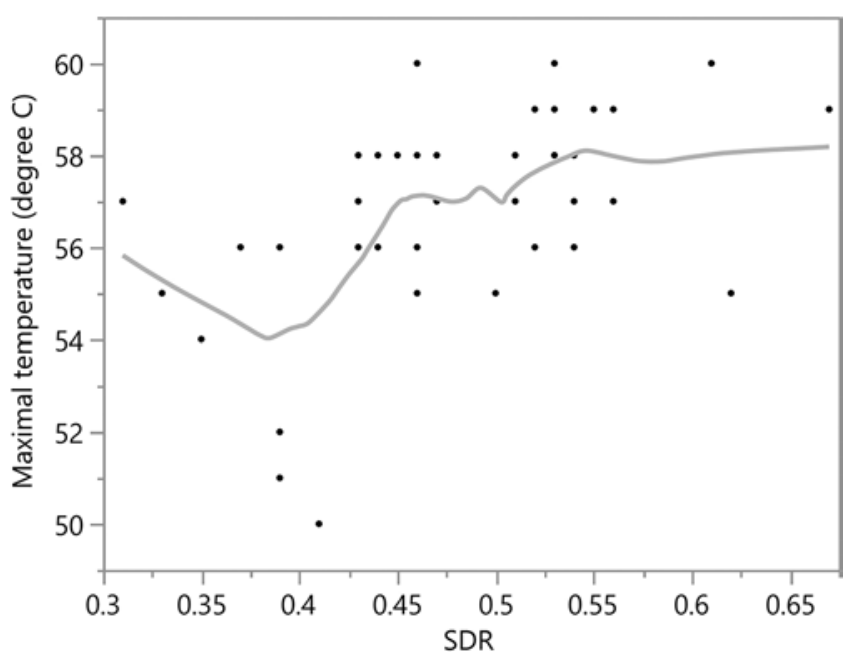

FIG. 4. Correlation of maximal temperature achieved during treatment and skull density ratio. A significant correlation was observed. The gray line is a LOWESS curve to illustrate the overall monotonic trend over most of the plot.

tremor at 12 months $(\varrho=0.49, \mathrm{p}=0.016)$, though it did not correlate with immediate outcome measures.

\section{Adverse Events}

All adverse events during and following the procedure were mild (Clavien-Dindo criteria for surgical interventions, grade 1; see Table 3). All adverse events appeared within the first week following the procedure, with no new adverse events during follow-up. Most adverse events were resolved within 3 months. The most common adverse events after the procedure were subjective unsteadiness and ataxia. The ataxia presented as gait ataxia and hand ataxia, usually within 1 day following the procedure. Adverse events after the procedure were reversible in all but 5 patients (11\% of patients) (Table 3). Unsteady gait

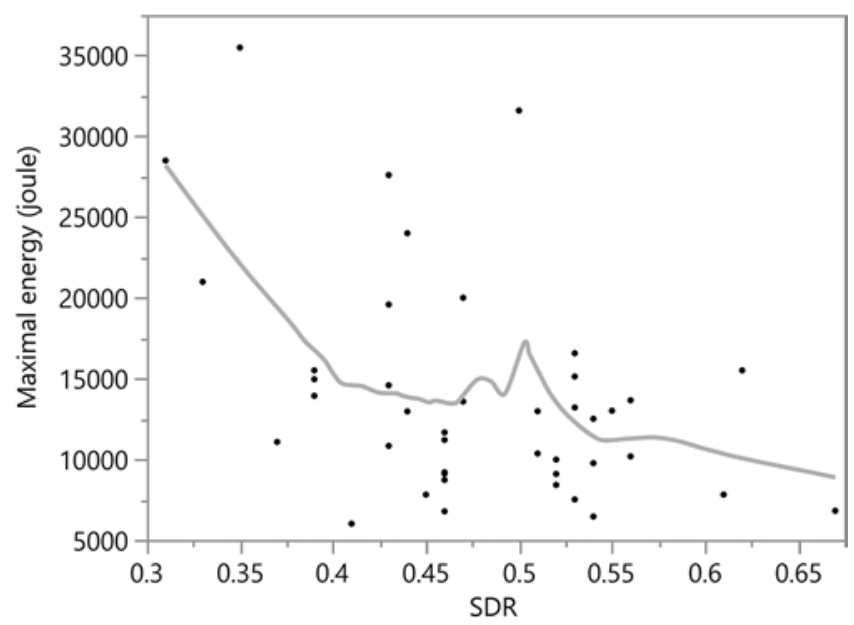

FIG. 5. Correlation of maximal energy achieved during treatment and skull density ratio. A significant correlation is observed. The gray line is a LOWESS curve to illustrate the overall monotonic trend over most of the plot.
TABLE 3. Adverse events

\begin{tabular}{lccc}
\hline \multicolumn{1}{c}{ Adverse Events } & $\begin{array}{c}\text { No. } \\
\text { of } \\
\text { Pts }\end{array}$ & $\begin{array}{c}\text { Resolved, No. } \\
\text { of Pts (time to } \\
\text { resolution in mos) }\end{array}$ & $\begin{array}{c}\text { Unresolved, No. } \\
\text { of Pts (follow-up } \\
\text { length in mos) }\end{array}$ \\
\hline During procedure & & & \\
\hline Vertigo & 23 & $23^{*}$ & 0 \\
\hline Headache & 17 & $17^{*}$ & 0 \\
\hline Nausea & 6 & $6^{*}$ & 0 \\
\hline Lip/tongue paresthesia & 6 & $6^{*}$ & 0 \\
\hline Head/scalp heat & 5 & $5^{*}$ & 0 \\
\hline Hand paresthesia & 4 & $4^{*}$ & 0 \\
\hline Vomiting & 2 & $2^{*}$ & 0 \\
\hline Dizziness & 2 & $2^{*}$ & 0 \\
\hline After procedure & & & $2(12)$ \\
\hline Gait ataxia & 10 & $8(1-3)$ & 0 \\
\hline Subjective unsteadiness & 8 & $8(0.25)$ & 0 \\
\hline Hand ataxia & 6 & $6(1)$ & $2(24)$ \\
\hline Dysgeusia/hypogeusia & 5 & $3(3)$ & 0 \\
\hline Asthenia & 4 & $4(1)$ & $2(12)$ \\
\hline Lip/tongue paresthesia & 4 & $2(3)$ & $1(6)$ \\
\hline Hand paresthesia & 2 & $1(1)$ & 0 \\
\hline Scalp numbness & 1 & $1(3)$ & \\
\hline Pts = patients. & & & \\
* Resolved by end of procedure. & & & \\
\hline
\end{tabular}

was significantly associated with skull volume $(\mathrm{p}=0.035)$ and skull area $(\mathrm{p}=0.038)$ and number of sonications $(\mathrm{p}=$ $0.046)$. Ataxia was also associated with skull volume ( $\mathrm{p}=$ 0.049 ) and marginally with skull area. Patients' age, sex, disease characteristics, and other treatment parameters did not significantly influence prevalence of adverse events.

\section{Discussion}

We report our experience in a single center of treating a cohort of $44 \mathrm{ET}$ patients with MRgFUS thalamotomy over a span of 5 years. We found that MRgFUS thalamotomy is an effective treatment for tremor relief, with all patients having significant decrease in tremor in the treated hand immediately following the treatment, with a significant decrease of CRST scores. In most patients, tremor relief was long lasting, even at 5-year follow-up. Quality of life was significantly correlated with tremor in the hand intended for treatment. Therefore, as expected, tremor relief translated into a significantly improved quality of life in most patients, as shown by a significant decrease of QUEST scores. Adverse effects of the procedure were mostly mild and reversible.

Tremor was relieved with MRgFUS thalamotomy in all patients spanning from middle age to very elderly patients. Patient sex as well as duration of tremor did not affect favorable results. The degree of tremor relief was $100 \%$ in the majority of patients, with consequent significant improvement in quality of life, and complete resolution of all burden on quality of life was reported by 7 patients (QUEST score of 0 ).

We sought to determine which treatment parameters 
and which patient characteristics predict outcome as measured by CRST and QUEST. We found that patient demographics, disease duration, and tremor scores did not correlate with outcome. Calculated SDR significantly correlated with outcome following treatment, but this parameter only correlated with outcome at 1 month and 6 months but not with outcome at 1 year and later. SDR also correlated with treatment parameters. We found significant correlation between SDR and maximal temperature reached at the end of the treatment and an inverse correlation between SDR and maximal energy reached during treatment. This is in line with the report of Chang et al., who found a significant correlation between SDR and maximal temperature reached. ${ }^{4}$ A possible explanation for these findings are that in order to achieve cessation of tremor in patients with lower SDR, significantly higher energy is required and even with higher energy the maximal temperature achieved at the VIM treatment target is lower. Another possible explanation is that since higher energy during sonication requires a longer cooling time after each sonication, in patients with lower SDR, for whom higher energy is used during treatments, intervals between sonications are longer. Thus, dynamic processes in the brain tissue during these intervals may affect tissue response to the following sonications. Particularly in the final sonications where high energy is used, intervals between sonications may reach up to 18 minutes, and tissue damage may preclude further response to sonications or may cause the appearance of cavitations in the tissue. These theories need to be explored and studied in order to allow treatment of patients with low SDR. It should be noted that Chang et al. ${ }^{4}$ reported a significant correlation between skull volume and maximal temperature, but in our cohort we did not replicate this finding. We found that maximal temperature was significantly associated with CRST scores, which meant that more severe tremor required a higher temperature in order to abolish the tremor.

We analyzed whether there is a threshold for successful treatment, since an SDR threshold was required for participation in studies looking at the efficacy and safety of this technology ( 0.45 in thalamotomy for ET study ${ }^{8}$ and thalamotomy for PD study'). We could not find such a threshold. On the contrary, we found that patients who experienced return of tremor during follow-up were the patients with slightly higher SDR parameters. Since few patients experienced return of tremor, it was not possible to correlate this phenomenon with treatment parameters or other demographic data.

One day after the procedure the volume of the edema around the lesion site was at its maximum. Consequently, the size of the lesion with its surrounding edema 1 day after the treatment was on average $1306 \mathrm{~mm}^{3}$. The size of the core lesion at its largest was seen after 1 week and was on average $392 \mathrm{~mm}^{3}$. The lesion size was significantly correlated with number of sonications, maximal sonication time, and maximal energy used during the sonication. Unexpectedly, we did not find a significant correlation between the lesion size with or without surrounding edema and total CRST scores after the procedure, in CRST scores in the treated hand, in Quest scores after the procedure, or in change of CRST and QUEST scores following the procedure.
Adverse events during the procedure were mild. ${ }^{7}$ All adverse events appeared within the first week following the procedure and in most patients resolved within 3 months. There were no new adverse events after the first week during follow-up. The most common adverse events after the procedure were unsteady gait and ataxia. During follow-up adverse events did not resolve in 5 patients (11\%). This adverse event profile is similar to that reported by Fishman et al., ${ }^{9}$ with serious adverse events reported in $1.6 \%$ of patients and most adverse events being transient and commonly rated as mild $(79 \%)$ and rarely as severe (1\%). Fishman et al. also reported that balance abnormalities and abnormal sensation were the most common, whereas we found that balance abnormalities were common but abnormal sensation occurred infrequently. Since the technology for MRgFUS thalamotomy is similar in all centers, the difference in the prevalence of abnormal sensation and other adverse events following the procedure in different centers may be attributed to differences in methodology. We have therefore suggested in the past that in order to avoid these adverse events MRgFUS thalamotomy should be performed in specialized centers. ${ }^{11}$

We found that the skull parameters, skull volume and skull area, significantly affected the occurrence of adverse events following treatment, but we found no association between SDR and the occurrence of adverse events. Furthermore, adverse events were not affected by age, sex, or disease duration or other treatment parameters.

As far as we know, this is the largest single-center cohort and longest follow-up duration reported for MRgFUS to date. However, with the number of parameters and variables included in the statistical analyses, the limited numbers of patients did not allow a covariate analysis to be done.

In addition, both the patients and the physicians were not blinded to the treatment; therefore, placebo effect and bias of the patient and the examining physician cannot be ruled out.

\section{Conclusions}

MRgFUS thalamotomy for ET is an effective and safe procedure that provides long-term tremor relief and improvement in quality of life even in patients with medication-resistant disabling tremor. Additional studies with a larger group of patients are needed to substantiate our favorable results.

\section{References}

1. Bond AE, Shah BB, Huss DS, Dallapiazza RF, Warren A, Harrison MB, et al: Safety and efficacy of focused ultrasound thalamotomy for patients with medication-refractory, tremordominant Parkinson disease: a randomized clinical trial. JAMA Neurol 74:1412-1418, 2017

2. Chang JW, Park CK, Lipsman N, Schwartz ML, Ghanouni $\mathrm{P}$, Henderson JM, et al: A prospective trial of magnetic resonance-guided focused ultrasound thalamotomy for essential tremor: results at the 2-year follow-up. Ann Neurol 83:107-114, 2018

3. Chang WS, Jung HH, Kweon EJ, Zadicario E, Rachmilevitch I, Chang JW: Unilateral magnetic resonance guided focused ultrasound thalamotomy for essential tremor: practices and 
clinicoradiological outcomes. J Neurol Neurosurg Psychiatry 86:257-264, 2015

4. Chang WS, Jung HH, Zadicario E, Rachmilevitch I, Tlusty T, Vitek S, et al: Factors associated with successful magnetic resonance-guided focused ultrasound treatment: efficiency of acoustic energy delivery through the skull. J Neurosurg 124:411-416, 2106, 2016

5. Chung AH, Jolesz FA, Hynynen K: Thermal dosimetry of a focused ultrasound beam in vivo by magnetic resonance imaging. Med Phys 26:2017-2026, 1999

6. Clement GT, Hynynen K: A non-invasive method for focusing ultrasound through the human skull. Phys Med Biol 47:1219-1236, 2002

7. Dindo D, Demartines N, Clavien PA: Classification of surgical complications: a new proposal with evaluation in a cohort of 6336 patients and results of a survey. Ann Surg 240:205213, 2004

8. Elias WJ, Lipsman N, Ondo WG, Ghanouni P, Kim YG, Lee $\mathrm{W}$, et al: A randomized trial of focused ultrasound thalamotomy for essential tremor. N Engl J Med 375:730-739, 2016

9. Fishman PS, Elias WJ, Ghanouni P, Gwinn R, Lipsman N, Schwartz M, et al: Neurological adverse event profile of magnetic resonance imaging-guided focused ultrasound thalamotomy for essential tremor. Mov Disord 33:843-847, 2018

10. Schlesinger I, Eran A, Sinai A, Erikh I, Nassar M, Goldsher $\mathrm{D}$, et al: MRI guided focused ultrasound thalamotomy for moderate-to-severe tremor in Parkinson's disease. Parkinsons Dis 2015:219149, 2015

11. Schlesinger I, Zaaroor M: A trial of focused ultrasound thalamotomy for essential tremor. N Engl J Med 375:2201-2202, 2016

12. Sinai A, Katz Y, Zaaroor M, Sandler O, Schlesinger I: The role of the anesthesiologist during magnetic resonanceguided focused ultrasound thalamotomy for tremor: a singlecenter experience. Parkinsons Dis 2018:9764807, 2018
13. Stacy MA, Elble RJ, Ondo WG, Wu SC, Hulihan J: Assessment of interrater and intrarater reliability of the FahnTolosa-Marin Tremor Rating Scale in essential tremor. Mov Disord 22:833-838, 2007

14. Sun J, Hynynen K: The potential of transskull ultrasound therapy and surgery using the maximum available skull surface area. J Acoust Soc Am 105:2519-2527, 1999

15. Tröster AI, Pahwa R, Fields JA, Tanner CM, Lyons KE: Quality of life in Essential Tremor Questionnaire (QUEST): development and initial validation. Parkinsonism Relat Disord 11:367-373, 2005

16. Zaaroor M, Sinai A, Goldsher D, Eran A, Nassar M, Schlesinger I: Magnetic resonance-guided focused ultrasound thalamotomy for tremor: a report of 30 Parkinson's disease and essential tremor cases. J Neurosurg 128:202-210, 2018

\section{Disclosures}

Menashe Zaaroor is a scientific consultant to Insightec.

\section{Author Contributions}

Conception and design: Schlesinger, Sinai, Nassar. Acquisition of data: Schlesinger, Sinai, Nassar, Constantinescu, Zaaroor. Analysis and interpretation of data: Schlesinger, Sinai, Nassar, Sprecher. Drafting the article: Schlesinger, Sinai, Nassar, Sprecher. Critically revising the article: all authors. Reviewed submitted version of manuscript: all authors. Approved the final version of the manuscript on behalf of all authors: Schlesinger. Statistical analysis: Schlesinger, Sprecher. Administrative/technical/material support: Schlesinger, Sinai. Study supervision: Schlesinger.

\section{Correspondence}

Ilana Schlesinger: Rambam Health Care Campus, Haifa, Israel. i_schles@rambam.health.gov.il. 